Ciencia y Educación, Vol. 4, No. 2, mayo-agosto, 2020

ISSN (impreso): 2613-8794 • ISSN (en línea): 2613-8808

DOI: https://doi.org/10.22206/cyed.2020.v4i2.pp75-86

\title{
Acoso escolar en un centro que implementa tutorías entre iguales
}

\author{
Bullying at a center that implements peer tutoring
}

\author{
Eva Linaje ${ }^{\mathrm{a}}$ ORCID: 0000-0002-7975-9065 \\ Almudena Cotán Fernández b ORCID: 0000-0002-7975-9065
}

Recibido: 7/02/2020 • Aprobado: 18/03/2020

Cómo citar: Linaje, E., \& Cotán Fernández, A. (2020). Acoso escolar en un centro que implementa tutorías entre iguales. Ciencia y Educación, 4(2), 75-86. Doi: https://doi.org/10.22206/cyed.2020.v4i2.pp75-86

\begin{abstract}
Resumen
En este artículo se presentan los resultados de un estudio cuantitativo que buscó caracterizar el acoso escolar en un centro educativo que cuenta con un programa de tutorías entre iguales. 205 alumnos de Educación Secundaria Obligatoria (11-16 años) participaron en el estudio. El instrumento de recogida de datos fue el cuestionario test Bull-S, y permitió analizar la relación entre el alumnado, las expectativas sociales, el nivel de cohesión social, lugares de ocurrencia del acoso escolar, entre otros. Los resultados obtenidos muestran que: (1) existe diversidad de actores y perfiles en el centro escolar estudiado; (2) el alumnado tiende al rechazo hacia el perfil de agresor; (3) el perfil del alumnado agresor evidencia altos niveles de popularidad y, (4) las principales formas de abuso identificadas son el insulto verbal, la amenaza y el rechazo.
\end{abstract}

Palabras clave: prevención; programas de educación; acoso; tutoría; convivencia.

\begin{abstract}
This article presents the results of a quantitative study that sought to characterize bullying in an educational center that has a peer tutoring program. 205 students from Compulsory Secondary Education (11-16 years old) participated in the study. The data collection instrument was the Bull-S test questionnaire, and allowed analyzing the relationship between students, social expectations, level of social cohesion, places of occurrence of bullying, among others. The results obtained show that: (1) there is a diversity of actors and profiles in the school studied; (2) students tend to reject the aggressor profile; (3) the profile of the attacking students shows high levels of popularity and, (4) the main forms of abuse identified are verbal insult, threat and rejection.
\end{abstract}

Keywords: Prevention; education program; bullying; coexistence; tutorships.

\footnotetext{
a Investigadora independiente, España. Correo-e: evalinaje@gmail.com

a Universidad de Cádiz, España. Correo-e: almudena.cotan@uca.es
} 


\section{Introducción}

Uno de los mayores desafíos en la educación actual es el acoso escolar o bullying. Este es considerado como una conducta de acoso repetitiva, intencionada y deliberada realizada por parte de un alumno hacia otro compańero que se encuentra en una situación de desprotección (Olweus, 1993; Sarasola y Ripoll, 2019). Este hecho supone numerosas situaciones estresantes para el alumnado que lo vive. Buena muestra de ello podemos encontrarlo en diferentes trabajos e informes como los de Cano-Echeverri y Vargas González (2018), Cerezo (2001, 2009, 2014, 2015), el Defensor del Pueblo (2007), Díaz, Martínez y Pérez (2017), Garaigordobil, Martínez-Valderrey y Machimbarrena (2014), González Bellido (2015), Musalem y Castro (2014), Ovejero (2010), Ovejero, Smith y Yubero (2013), Solberg, Olweus y Endresen (2007) o Puértolas y Montiel (2017). Estos trabajos evidencian que las variables acoso escolar y estrés están íntimamente relacionadas. Este hecho se hace especialmente visible en el alumnado presentando cuadros de ansiedad, tristeza, soledad, deterioro de la autoestima o depresión, entre otros (Cano-Echeverri y Vargas González, 2018; Cerezo, 2015; Díaz et al., 2017; García y Asensio, 2015; Sarasola y Ripoll, 2019; Vanega-Romero, Sosa-Correo y Castillo-Ayuso, 2018).

Los informes sobre acoso escolar realizados por la Organización de Naciones Unidas para la Educación, la Ciencia y la Cultura (UNESCO, 2018) indican que, a nivel mundial, uno de cada tres adolescentes sufre acoso escolar. Para la obtención de estos datos, recogidos entre 2014 y 2016, la UNESCO (2018) utilizó dos estudios: 1. Encuesta Mundial de la Salud a Escolares centrada en alumnos de entre 13 y 17 años en regiones con pocos ingresos y, 2. Estudio sobre Conductas de los Escolares relacionadas con la Salud que se centra en alumnos de entre 11 y 15 ańos de 42 países, principalmente, de Europa y América del Norte. Ambos estudios evidencian una realidad llamativa: existe un amplio colectivo de alumnado que ha sufrido acoso escolar en los últimos 12 meses. Entre los países con menos recursos, se encuentran cifras realmente alarmantes: el 46,6 \% en Namibia y el 44 \% en Afganistán de los escolares, sufren acoso escolar. Sin embargo, también hay países europeos con un alto índice de acoso escolar como es el caso de Lituania, con un 53,98 \%, Letonia con un 49,67 \%, Hungría con un 30,77 e Irlanda con un $27,6 \%$. Cifras demasiado altas que reflejan un hecho importante: el acoso escolar es un problema latente a nivel mundial.

En Espańa se encuentran datos similares a los ya obtenidos por Olweus (1993), donde cerca del 15\% de la población escolarizada entre los 11 y 16 ańos aseguraron haber estado implicados en alguna situación de acoso. Datos similares a los identificados por Cerezo y Méndez (2012, 2013), Cerezo, Sánchez, Ruiz y Arense (2015), García, Pérez y Nebot (2010) y Vieira, Fernández y Quevedo (1989), donde aproximadamente el $20 \%$ de la población estudiantil ha vivido alguna situación de acoso escolar.

Otros informes oficiales, como el Informe Cisneros $x$ (Oñate y Piñuel, 2007), el Informe sobre Violencia Escolar del Defensor del Pueblo (2007), los informes de Gobierno Vasco de 2012 sobre el maltrato entre iguales en Primaria y Educación Secundaria Obligatoria, el informe sobre violencia entre iguales (2016) o el Estudio Estatal sobre la Violencia Escolar en Educación Secundaria Obligatoria (Díaz, Martínez y Martín, 2010), reflejan y constatan esta realidad. Las principales medidas preventivas a adoptar en esos informes son coincidentes: existe una necesidad imperante de combatir este problema desde los centros educativos y las administraciones públicas pertinentes. Para ello, campañas de sensibilización, prevención y formación son necesarias.

Derivado de esto, son numerosas las medidas que han surgido para combatir el acoso escolar. A nivel internacional cabe destacar los programas Olweus Bullying Prevention Program (OBPP), el Programa Zero en Noruega, el Programa KiVa en Finlandia o el Programa Be-Prox en Suiza. Desde estos programas se pretende prevenir el acoso escolar y promover valores de tolerancia cero. Para ello, buscan la implicación de toda la comunidad educativa, no solo de víctimas y agresores en el programa, sino también de los testigos, ya que, según indica Mäkelä (2017), al agresor no le resulta tan divertido el acoso si no se ve apoyado por los testigos. En el caso del programa Zero noruego también se involucra a padres y educadores, además del grupo de pares. En Reino Unido se desarrolló el 
programa KIDSCAPE dirigido a niños de temprana edad (de 3 a 11 años) con el objetivo de protegerles ante el acoso escolar o el abuso sexual, entre otros.

Similar a esta acción, en España se encuentra el programa Tutoría entre iguales (TEI). TEI, creado por González-Bellido (2002), comienza a implementarse en centros de primaria y secundaria en el curso 2003-2004. Se centra en un programa de convivencia que previene la violencia y el acoso escolar implicando a toda la comunidad educativa. Influenciado por las teorías de los Sistemas Ecológicos (Bronfenbrenner, 1987), la Inteligencia y Educación Emocional (Bisquerra, 2000; Goleman, 1995; Salovey y Mayer, 1990) y la Psicología Positiva y Optimista (Seligman, 1990), el programa TEI busca fomentar relaciones entre iguales satisfactorias en las que impere el respeto y la tolerancia cero a la violencia y el acoso escolar. Fomenta el desarrollo de herramientas resilientes para la resolución de conflictos entre los alumnos, siendo estos los principales elementos de dinamización del programa. Su objetivo es mejorar la integración escolar y trabajar para conseguir un ambiente escolar inclusivo y no violento.

Estudios como los de Albaladejo, Caruana, González, Giménez, Ferrer y Sánchez (2016) y Ferrer, Albaladejo, Sánchez, Portilla, Jordan y Ruiz (2019), reflejan los beneficios de dicho programa. Los resultados obtenidos son concisos: 1. Reducción significativa de comportamientos de acoso escolar y también en el ciberacoso (Giménez, Arnaiz, Cerezo, y Prodócimo, 2018) y, 2. Mejora en el clima escolar. En este orden de ideas, Andrés González, diseñador del programa TEI, en una entrevista realizada por la Universitat de Barcelona (2017) asegura que "con el programa TEI, desparece en un $95 \%$ el acoso escolar, se minimiza la victimización y disminuye el nivel de estrés y de ansiedad" (encabezado).

\section{Metodología}

Este artículo parte de un trabajo de investigación realizado en el Máster Universitario del Profesorado (MAES) de la Universidad de Nebrija durante el curso 2018/2019. El objetivo principal del estudio era caracterizar la realidad del acoso escolar en un centro educativo del País Vasco (Espańa) que cuenta con un programa de TEI.

La metodología seleccionada para tal fin fue la cuantitativa. A través de la misma se realizó un estudio sociométrico de los principales componentes (alumnos de ESO) y del nivel de cohesión existente en el grupo. También se evaluaron condiciones socio-afectivas (como son vistos por sus compañeros y por sí mismos) entre agresor y víctima y los contextos en los que tenían lugar las agresiones. De esta forma, tres fueron los principales criterios de análisis: posición social del alumno; aceptación-rechazo, y, agresividad-victimización.

El instrumento de recogida de información utilizado ha sido el test Bull-s creado por Cerezo en el año 2000 (actualizado en 2012). El test Bull-s está diseñado para medir la agresividad entre escolares, estando dirigido no solo a agresor y víctima, sino a todos los elementos personales que forman el aula, es decir incluye también a los testigos, los profesores y demás empleados del centro, así como a las familias, implicando, de este modo, a toda la comunidad educativa. Dicho instrumento quedó validado a través de la propia autora (Cerezo, 2009). Tal y como indican Méndez y Cerezo (2010, p. 3), su "validez viene determinada por la precisión con la cual el instrumento mide el índice de agresividad entre los escolares, la configuración del grupo y su respuesta ante esta problemática, así como los aspectos situacionales analizados".

El principal motivo de la selección de este instrumento reside en el objetivo principal del mismo: permite analizar y comprender el problema del acoso escolar, tanto individual como colectivamente, y facilita la intervención psicopedagógica. Esta herramienta ofrece la posibilidad de recoger información a través de dos vías: 1 . Directa con cuestionarios a los alumnos $y, 2$. Indirecta con cuestionario a los profesores. En este estudio únicamente se utilizará la primera vía: directa con cuestionarios dirigidos a alumnos. El cuestionario dirigido al alumnado recoge 15 preguntas agrupadas en tres bloques:

1. Primer bloque: posición social del alumno en el grupo y nivel socio afectivo del grupo (preguntas 1-4). Se pregunta sobre los alumnos 
a los que elegirían para sentarse en clase y con quiénes no quieren sentarse, así como quiénes creen que querrían sentarse con ellos y quiénes creen que los rechazarían.

2. Segundo bloque: relación agresión-victimización (preguntas 5-10). Se plantean preguntas para ver, en opinión de los alumnos, quiénes son vistos como fuertes, cobardes, agresivos, víctimas, provocadores o a quiénes se les tiene manía en clase.

3. Tercer bloque: analiza los lugares en los que más asiduidad se producen las agresiones y la valoración de la gravedad de las mismas (preguntas 11-15). Hace especial énfasis en la forma, lugar, frecuencia y gravedad de las agresiones, así como en su percepción de seguridad en el centro.

El cuestionario fue facilitado en todas las aulas de secundaria (dos líneas por curso), más una línea para el grupo de Proyecto de Refuerzo Educativo Específico (PREE) al que acuden alumnos con necesidades especiales educativas de los cursos primero y segundo de ESO y otra para Diversificación Curricular (DIVER) para alumnos con necesidades especiales educativas de tercero y cuarto de ESO. Obteniendo un total de 10 aulas y 209 alumnos de ESO con edades comprendidas entre los 11 y los 16 ańos.

Los cuestionarios fueron pasados dentro del horario lectivo, junto con el tutor de grupo de referencia. La duración del mismo osciló entre 15 o 20 minutos. Hubo una participación del $98 \%$. El 2 \% restante estaba ausente o rechazó su realización. Referente al alumnado con alguna necesidad educativa, no solicitó ningún tipo de adaptación de contenido. Sin embargo, sí que fueron necesarias orientaciones y aclaraciones sobre su realización.

El análisis de los resultados será comparativo entre los diferentes cursos. Se realizará teniendo en cuenta las distintas dimensiones que abarca el test Bull-S: Sociométrica (preguntas 1-4), dinámica de acoso escolar (preguntas 5-10), y situacionales (preguntas 11-15). Este análisis se realizará atendiendo a las indicaciones del "Manual Test Bull-S versión 2.2" (Cerezo, 2012) y con ayuda del programa informático que ofrece el propio test (Méndez y Cerezo, 2010).

\section{Contexto y participantes}

La investigación se desarrolló en un colegio privado situado en el norte de España. Cuenta con las etapas de Educación Infantil (0-6 años), Educación Primaria (6-12 años) y Educación Secundaria (12-16 años), y una población de 712 alumnos. En este trabajo solo se han analizado los datos procedentes del alumnado de Educación Secundaria Obligatoria (ESO) y las aulas de PRE y DIVER. Se trabajó con un muestreo no probabilístico e intencional.

Para acceder a la muestra, se solicitó previamente el consentimiento de los padres y tutores legales del alumno. Para ello, a través del jefe de estudio y del psicólogo del centro, fueron informados del programa TEI y sus objetivos, en una reunión. Posterior a ello, a través de los tutores y tutoras de los grupos de referencia se procedió a facilitar la información del estudio al alumnado, así como el instrumento de recogida de datos.

Con una muestra inicial de 209 alumnos en la etapa de secundaria y cursos, PRE y DIVER, en el estudio finalmente participaron un total de 205 alumnos (tres alumnos se encontraban ausentes y uno se negó a realizar el cuestionario). De estos 205 alumnos, 115 son de sexo masculino y 90 de sexo femenino, oscilando sus edades entre los 11 y los 16 años. Lo que supone un $98 \%$ de la población total de estudiantes de estos cursos. El 2 \% restante no participó en el estudio por varios motivos: ausencia, enfermedad o no voluntariedad para su realización.

Atendiendo a la Ley Orgánica, 3/2018, de 5 de diciembre, de Protección de Datos Personales y garantía de derechos digitales, para evitar la identificación de los estudiantes, en el análisis y difusión de los resultados, sus nombres reales fueron sustituidos por un código numérico. En todo momento, se ha asegurado y preservado la confidencialidad de los datos obtenidos, así como la identidad de los menores.

\section{Resultados}

Los resultados obtenidos se presentan en tres grandes bloques: 1. Variables relativas a la aceptación/rechazo en las que se analizará la relación del alumnado en 
clase, las expectativas sociales, el nivel de cohesión grupal y el clima del aula; 2 . Variables referentes a la dinámica de acoso escolar en la que se evaluará el perfil del alumnado, la incidencia del acoso y la repercusión del mismo y, 3. Variables situacionales en las que se identificarán las principales formas y lugares donde tiene lugar el acoso así como la topografía y la seguridad identificada por parte del alumnado en el centro escolar.

\section{a) Variables relativas a la aceptación/rechazo}

En este apartado se presentan los resultados sociométricos que permiten conocer el tipo de relación que existe entre los alumnos, sus expectativas de popularidad y de ser rechazados, así como la estructura del grupo y el nivel de cohesión existente.

\section{Posición sociométrica (preguntas 1-4)}

\section{Relaciones de los alumnos en clase (preguntas 1 y 2)}

Referente a las relaciones que el grupo de alumno mantiene en clase, cinco fueron las opciones que se le ofrecieron: popular (alumno considerado popular y conocido entre sus compańeros), aislado (alumno que no se incluye en ningún grupo), rechazado (alumno que no es aceptado por el grupo de pares), controvertido (tienen un cierto grado de popularidad para un sector del grupo y son rechazados por otro sector del grupo), promedio (no se encuentran en ninguna de las cuatro categorías anteriores). En los datos obtenidos, se observa que el $44 \%$ del alumnado considera que suele participar e interactuar de manera asidua con sus compañeros. Le siguen con un 25,6 \% el alumnado que se considera controvertido y el alumnado que se considera aislado con un 14,8\%. En las dos últimas posiciones se sitúan el perfil rechazado con un $10 \%$ y el perfil popular con un 4,3\%. El 1,3\% del alumnado decidió no responder a esta cuestión ya que se negaron a clasificar de este modo a sus compañeros o no creían que se daban estas opciones.

Expectativas sociales de los alumnos (preguntas 3 y 4)

En la gráfica uno, se analizan las expectativas de popularidad del alumnado, lo que alumno piensa de sí mismo (socio-matriz 3) comparadas con las elecciones realizadas por sus compañeros, lo que alumnado piensa de sus compañeros (socio-matriz 1). Los resultados muestran porcentajes dispares en función del aula evaluada. Esto se torna especialmente visible en los grupos de $2^{\circ} \mathrm{A}, 3^{\circ} \mathrm{B}$ y DIVER, donde predominan los alumnos no sociables, mientras que, en el resto de aulas, el porcentaje mayor lo ocupa el alumnado que se identifica como sociable. De esta forma, obtenemos que el 40,2 \% del alumnado se considera sociable (alta expectativa de ser elegido por sus compañeros por sus grandes habilidades sociales para relacionarse con ellos y con los profesores) frente al 27,3\% que se considera equilibrado y el 31,1\% no sociable (considera que no será elegido por sus compañeros por carecer de habilidades sociales).

\section{EXPECTATIVA DE POPULARIDAD}

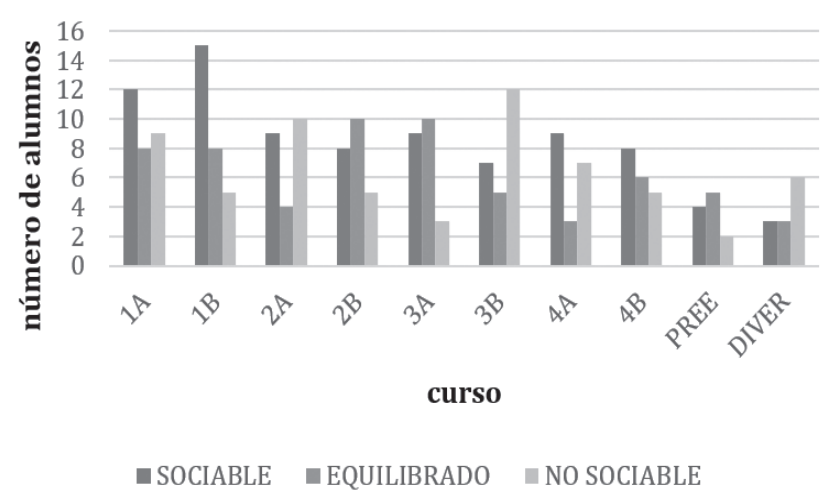

Gráfico 1. Expectativa de popularidad

Fuente: elaboración propia.

En la segunda gráfica, se presenta una combinación de los datos de precepción de rechazo de los alumnos (socio-matriz 4) y los alumnos rechazados por sus compañeros (socio-matriz 2). Pretende comprobar si la percepción del alumno se corresponde con la percepción de sus compañeros. En ella se refleja una predominancia clara respecto a las expectativas de rechazo bajas $(55,9 \%)$, mientras que un $23,9 \%$ tiene expectativas altas de ser rechazado. 


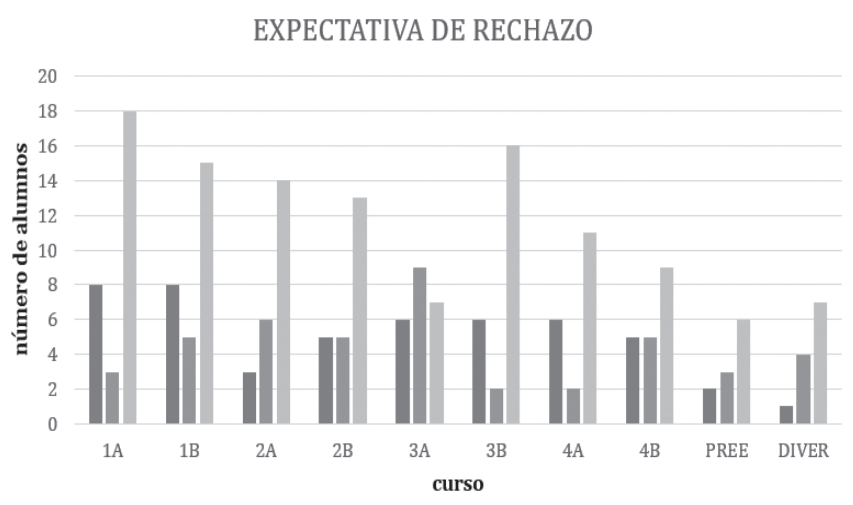

Gráfico 2. Expectativas de rechazo

Fuente: elaboración propia.

\section{Estructura del grupo}

En este gráfico se puede apreciar la estructura del grupo. Se observa que en todas las aulas los alumnos se agrupan entre sí formando diferentes grupos cohesionados, salvo en DIVER, donde se encuentra un nivel bajo de cohesión (16\%). Este dato muestra una menor sociabilidad de sus integrantes. Referente a la formación de los grupos, se determina que varios alumnos aparecen en diversos grupos. Estos alumnos son identificados como equilibrados y sociables (gráfico 3).

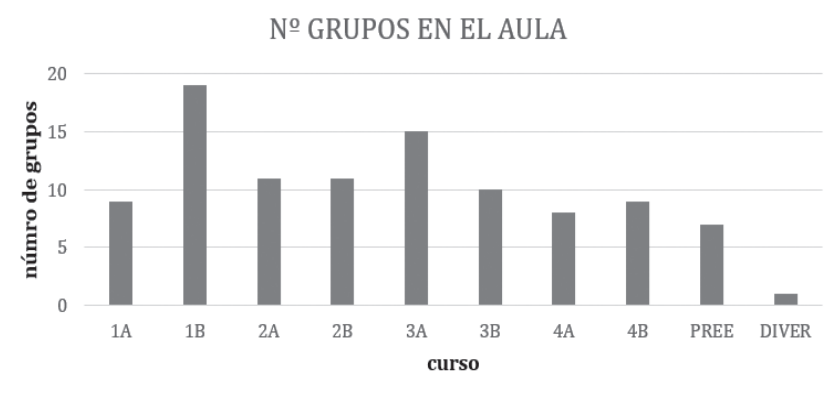

Gráfico 3. Número de grupos en el aula

Fuente: elaboración propia.

\section{Nivel de cohesión}

Los datos obtenidos evidencia que en las aulas no se aprecian niveles altos de cohesión. En seis de las diez aulas se da un nivel bajo de cohesión. Sin embargo, en muchas de ellas el porcentaje alcanzado se acerca al nivel óptimo: un 44,2\% del alumnado refleja niveles de cohesión. Sin embargo, se observa que el nivel de cohesión es muy baja en el aula de DIVER (16 \%).

\section{b) Variables relativas a la dinámica de acoso escolar (preguntas 5 a 10)}

En este apartado se busca detectar las situaciones de abuso, las características de los actores y la representación social de estos en el grupo. Las preguntas 5 a 10 del cuestionario darán la clave sobre los elementos de la relación agresión-victimización entre los alumnos del centro. Se incluyen los resultados de estas preguntas por cursos para, posteriormente, concretar la incidencia y la repercusión de la dinámica agresor-víctima.

De esta forma, se puede indicar que el alumnado presenta cierta diversidad de perfiles en función del curso. En general, se puede afirmar que no abundan los perfiles de agresores ni de víctimas. Solo en el aula $4^{\circ} \mathrm{B}$ destaca con un mayor porcentaje las víctimas percibidas $(21 \%)$. Los alumnos más provocadores parecen ubicarse en $1^{\circ} \mathrm{B}$ y PREE mientras que los más fuertes en $4^{\circ} \mathrm{A}$ y $\mathrm{B}$.

\section{Incidencia agresor-victima en el grupo}

En esta variable del cuestionario, se trata de detectar la presencia de agresores y víctimas, así como comprobar qué variables se asocian a su perfil. En la mayoría de las ocasiones el perfil de agresor coincide con el de provocador y en algunas ocasiones con el de fuerte, mientras que el perfil de víctima coincide con el de cobarde y el de se tiene manía.

Por otro lado, resalta el caso de $2^{\circ} \mathrm{B}$ en el que no se encuentran perfiles de agresor ni de víctima y los cursos $3^{\circ} \mathrm{B}$ y $4^{\circ} \mathrm{A}$ donde solo encontramos un perfil de agresor y no muy definido, al ser nominado por un $25 \%$ de los alumnos, porcentaje límite a partir del cual se considera a un alumno como agresor. El porcentaje de agresores es de un 5,7 \% y el de víctimas de un 6,7 \% respecto al total de alumnos. Los resultados obtenidos en este trabajo revelan que, en este centro escolar, un 5,3\% del alumnado de ESO tiene perfil provocador (tan solo 11 alumnos) lo que, a tenor de los resultados expuestos, se puede indicar que este perfil tiene una predominancia baja en el centro escolar. Asimismo, este perfil, provocador, el 
alumnado lo asocia e identifica más con el perfil de agresor (10) que con el de víctima (1).

Repercusión que la dinámica agresor-victima tiene en el grupo a nivel individual y grupal

A nivel individual se aprecia que el conjunto del grupo percibe más positivamente al agresor, quién, en muchas ocasiones, también es calificado como fuerte (50\% de los agresores) y sería elegido por sus compańeros (socio-matriz 1 y 3 ), mientras que, los perfiles de víctima son peor valorados. Se les identifica como cobardes ( $75 \%$ de las víctimas) y con importantes resultados de rechazo (socio-matriz 2 y 4 ).

A nivel grupal, se aprecia que el $44 \%$ de los agresores forman parte de grupos (alumnos considerados equilibrados en un $60 \%$ de los casos, e incluso uno popular), no así las víctimas (alumnos aislados en todos los casos). Por su parte los niveles de rechazo para los agresores son altos o muy altos (cercanos al 90 \%) salvo en PREE, DIVER y $4^{\circ} \mathrm{A}$ donde se considera a estos alumnos con perfil de agresor equilibrados o no rechazados. Las víctimas, sin embargo, no son consideradas como alumnos rechazados, salvo en los cursos $1^{\circ} \mathrm{A}, 1^{\circ} \mathrm{B}$, PREE y $4^{\circ} \mathrm{B}$.

Respecto a la formación de grupos en clase, en siete de los diez cursos ninguno de los dos perfiles forma parte de un grupo, mientras que en $4^{\circ} \mathrm{B}$ y $3^{\circ} \mathrm{A}$ se localizan perfiles de agresores y perfiles de víctimas en la formación de grupos. En $1^{\circ} \mathrm{B}$ la víctima es aislada y el agresor sí forma parte de grupos.

\section{c) Variables situacionales (preguntas 11-15)}

En este apartado, se examinarán cuáles son las agresiones más frecuentes, en qué lugares son ejecutadas, con qué frecuencia y qué importancia conceden los alumnos a estos posibles casos de acoso. También se analizarán el nivel de seguridad que percibe el alumnado en el centro escolar.

\section{Aspectos situacionales}

En el gráfico cuarto se refleja la forma más frecuente de agresión, siendo el insulto y la amenaza el más utilizado (45,2 \%), seguido del rechazo (44\%) y la agresión física $(10,8 \%)$. El lugar en el que con más frecuencia se produce el acoso escolar es en el aula, seguido del patio (escenario más frecuente en alumnos de primaria), mientras que en los pasillos apenas se producen agresiones.

Respecto a la frecuencia de las agresiones, la mayoría de los alumnos considera que se producen rara vez, únicamente los alumnos de PREE indican que se producen una o dos veces por semana, y los de $2^{\circ} \mathrm{A}$ indican porcentajes semejantes de respuesta para las opciones "todos los días", "una o dos veces por semana" y "raramente".

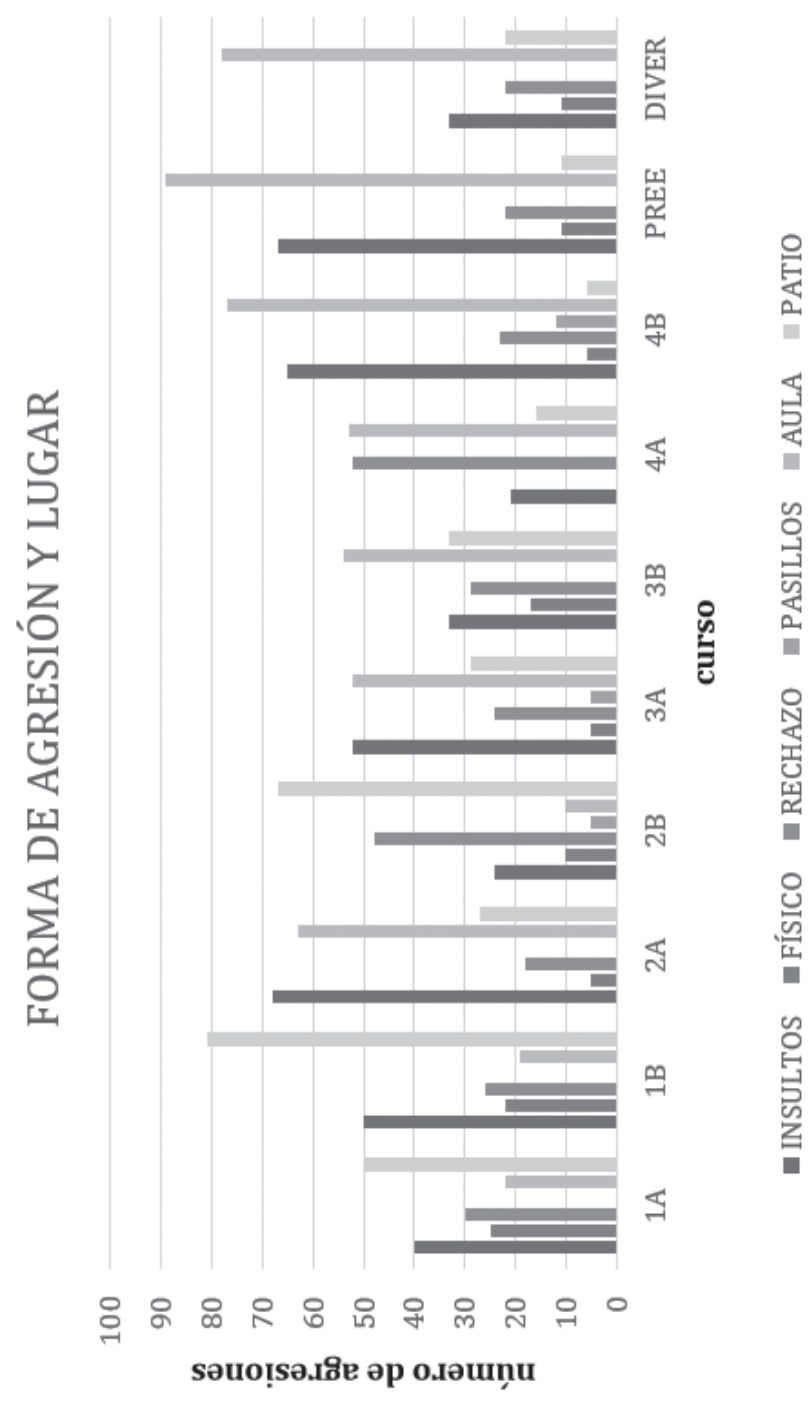

Gráfico 4. Formas de agresión y lugar

Fuente: elaboración propia. 


\section{Topografia y seguridad}

Este apartado permite ver que el alumnado otorga poca importancia a las agresiones que se producen en el centro escolar (el 40,75\% de los alumnos le da poca importancia, el 34,5\% moderada, el 13,5\% bastante y el 8,25\% mucha importancia) y que, en su mayoría, se sienten muy seguros en el mismo (un 1,5\% se siente poco seguro, un $14 \%$ regular, un $34,5 \%$ bastante y un $50 \%$ muy seguro). De nuevo, se observa que es el curso $1^{\circ} \mathrm{B}$ de ESO en el que mayor importancia da a las agresiones $(22,5 \%$ le da mucha importancia, el 29,3\% bastante, el 29,2 \% regular y el $19 \%$ poca importancia) y el que menos seguro se siente en el centro escolar (el $11 \%$ se siente poco seguro, el $26 \%$ regular, el $30 \%$ bastante y el $33 \%$ muy seguro), aun así, siguen siendo porcentajes de sentimiento de seguridad bastante altos.

\section{Conclusiones}

Partiendo de la realidad social del problema del acoso escolar, las instituciones públicas y educativas se plantean la búsqueda de soluciones eficaces y efectivas para su prevención. El objetivo principal de este trabajo fue analizar el estado del acoso escolar en un centro educativo del País Vasco (España) que cuenta con un programa de Tutorías Entre Iguales (TEI). Los resultados del estudio determinan que aproximadamente tres cuartas partes del alumnado de ESO de este centro escolar, es descrito por el perfil de alumno promedio o controvertido, y que solamente una doceava parte del alumnado es considerado popular. Asimismo, una sexta parte de este alumnado encaja en el perfil de alumno aislado, y menos de uno de cada veinte alumnos encaja en el perfil alumno rechazado (el $2 \%$ restante se corresponde con los alumnos que no han contestado los cuestionarios por estar ausentes, salvo un alumno de PREE que se negó a contestar el cuestionario).

Si se contrastan estos datos con los obtenidos en el segundo bloque de preguntas del cuestionario, se puede indicar que la mayoría del alumnado se ubica en el papel de observadores, mientras que el porcentaje de posibles agresores y el de posibles víctimas se situarían en porcentajes inferiores a los establecidos por Cerezo (2014). Asimismo, el informe Cisneros $\mathrm{x}$ (Ońate y Piñuel, 2007) indica que un 23,2 \% de los alumnos en alguna ocasión se ha considerado víctima de acoso. Por lo que, a tenor de lo expuesto, se puede concluir que este colegio actualmente tiene unos niveles de acoso escolar inferiores a la media de otros estudios. A su vez, si atendemos a los resultados ofrecidos por autores como Martínez (2017), se puede indicar que los resultados son muy alentadores, ya que en su estudio se reflejan cifras más altas (18,8\%). En este trabajo se identifica el acoso verbal como la forma más asidua de acoso. Dato que también se verifica en el informe de Martínez (2017) para el Gobierno Vasco. Referente al perfil del alumnado agresor, suele coincidir con el de provocador y fuerte en muchas ocasiones, mientras que el de víctima lo hace con el "cobarde". Respecto a este dato se asocian coincidencias con los patrones observados por Cano-Echeverri y Vargas González (2018), Cerezo y Méndez (2013), Díaz et al., (2017) o Rodríguez y Mejía (2012).

Estudios consultados (Cerezo, 2014; Cerezo et al., 2015; Giménez et al., 2018; Informe del Defensor de Pueblo, 2007) evidencian que es mayor la popularidad y percepción positiva sobre los alumnos agresores que sobre las víctimas, a las que se rechaza y se ve como cobardes. Sin embargo, los resultados obtenidos en este estudio indican que este hecho no se reitera en todos los cursos: existe un gran rechazo de los agresores en la mayoría de las aulas (siete de diez) y no se considera a las víctimas como aisladas ni rechazadas. De esta forma, se evidencia que en el centro educativo de estudio existen valores como la sensibilización y la tolerancia cero ante situaciones de acoso por parte del alumnado.

En este sentido, Arregi y Martínez (2012) en su informe para el Gobierno Vasco, coinciden con estos datos, indicando que ha aumentado la sensibilidad de las aulas en la toma de conciencia frente a las situaciones que genera el acoso escolar gracias a la implementación progresiva de programas de prevención. Entre los cursos en que se considera que las víctimas son rechazadas se encuentran los dos cursos de primero de ESO y PREE, este resultado concuerda con los presentados por otros autores anteriormente mencionados, destacando la mayor incidencia de tasas de abuso en esos cursos (Martínez, 2017). Asimismo, en 
estos cursos (primero de ESO y PREE) se observa que los alumnos vistos como agresores forman parte de los grupos formados en el aula, mientras que las calificadas como víctimas son excluidas, datos que se corresponden con los presentados por Cerezo (2014, 2015), Giménez et al., (2018) y Sánchez y Cerezo (2011). Por otro lado, García y Ascensio (2015) matizan que en el centro escolar no suele encontrarse el origen de las situaciones de acoso, pero puede favorecerlas o incluso perpetuarlas. Por otro lado, se coincide con autores como Ovejero et al., (2013) cuando afirman que, en los centros escolares más sensibilizados contra el acoso escolar se dan menos casos de maltrato.

Respecto a los niveles de sociabilidad de los alumnos, los resultados varían de unos cursos a otros, predominando los alumnos sociables en siete de los diez grupos. Los grupos con resultados no sociables son las aulas $2^{\circ} \mathrm{A}, 3^{\circ} \mathrm{B}$ y DIVER, de las que $2^{\circ} \mathrm{A}$ y DIVER también coinciden con los porcentajes más bajos de cohesión. En principio, parece lógico que estos cursos también deberían ser los que mayor expectativa de rechazo y en los que más posibilidades de acoso escolar pueden darse, sin embargo, los resultados obtenidos nos indican que los niveles de expectativas de rechazo son bajos para todos los cursos y no parece que haya mayor incidencia de acoso entre ellos. Los datos presentados avalan que, salvo los alumnos de DIVER, todos los alumnos de ESO tienen las suficientes habilidades sociales como para formar grupo y, en general, los niveles de cohesión son cercanos al óptimo, de lo que se deduce que, en la mayoría de los cursos de secundaria, el clima de la clase es bueno y las relaciones entre los iguales son fluidas. Ahondando más en esta idea, se observa que los niveles de percepción de seguridad de los alumnos en el centro son muy altos, cercanos al $100 \%$ y que su percepción sobre la importancia de las agresiones que se dan el centro escolar es baja o muy baja (siempre inferior al $50 \%$ ). Respecto a los aspectos situacionales, el lugar donde mayores agresiones se producen es el aula, seguido del patio. Valores coincidentes con los expuestos por autores (Arregi y Martínez, 2012; Martínez, 2017; Ońate y Piñuel, 2007).

Otra de las conclusiones obtenidas es la referida a la importancia y el nivel de seguridad del alumnado frente al acoso escolar. Respecto a los alumnos de $1^{\circ} \mathrm{ESO}$, son los que mayor importancia dan a las situaciones de acoso escolar y dicen sentirse menos seguros, si comparamos los datos con los del resto de cursos, su nivel de seguridad percibido sigue siendo muy alto. El informe del Defensor del Pueblo (2007) también apunta que es la franja de edad correspondiente a este curso (12-13 años) presenta mayor incidencia en conductas de acoso. Sin embargo, si se ponen en relación las respuestas obtenidas para los alumnos de $1^{\circ} \mathrm{ESO}$ en todas las preguntas del bloque III, se observa que, si bien son los alumnos que más importancia dan a las agresiones, también opinan que tienen lugar rara vez, por lo que el dato relativo a la importancia de las agresiones puede deberse a una mala interpretación de la pregunta 14 del cuestionario.

Para finalizar, los resultados obtenidos sugieren que la implementación de programas que prevengan el acoso escolar es positiva (Sarasola y Ripoll, 2019). A través del mismo, se genera una cultura de convivencia pacífica que fomenta la educación emocional y las buenas relaciones con los iguales basándose en el principio de tolerancia cero al acoso. Al igual que el estudio de Ferrer et al., (2019), y dado los resultados objetivos en este trabajo, se sugiere elaborar futuros estudios que profundicen en la efectividad de los Programas de Tutoría entre Iguales (TEI) para prevenir y reducir la violencia escolar y mejorar el clima social de las aulas.

\section{Referencias}

Albaladejo, N., Caruana, A., González, A., Giménez, P., Ferrrer, R. y Sánchez, M. (2016). Evaluación del programa Tutoría Entre Iguales (TEI). En J.L. Castejón, Psicología y Educación: Presente y Futuro (pp. 1636-1645). España: Asociación Científica de Psicología y Educación (ACIPE).

Arregi, A. y Martínez, P. (2012). El maltrato entre iguales en educación primaria y ESO Euskadi. Informe ejecutivo 2012. IESI.IVEI Eusko Jaurlaritza Gobierno Vasco Departamento de Educación, Universidad e Investigación. Bilbao. Disponible en http://www.bienestaryproteccioninfantil.es/fuentes 1. asp?sec $=27$ ycod $=2374$ 
Bisquerra, R. (2000) Educación emocional y bienestar. Madrid: Wolters Klumer Educación.

Boletín Oficial del Estado (2018). Ley Orgánica, 3/2018, de 5 de diciembre, de Protección de Datos Personales y garantía de derechos digitales. Disponible en https://www.boe.es/buscar/doc.php? $\mathrm{id}=$ BOE-A-2018-16673

Bronfenbrenner, U. (1987). La Ecología del Desarrollo Humano. Buenos Aires: Editorial Paidós.

Cano, M.M. y Vargas, J.E. (2018). Actores del acoso escolar. Revista Médica Risaralda, 24(1), 61-63. Doi: http://dx.doi.org/10. 22517/25395203.14221

Cerezo, F. (2001). Variables de personalidad asociadas en la dinámica bullying (agresores versus víctimas) en niños y niñas de $10 \mathrm{a}$ 15 años. Anales de Psicología, 17(1), 37-43. Disponible en https://www.um.es/analesps/ v17/v17_1/04-17_1.pdf

Cerezo, F. (2009). Bullying: análisis de la situación en las aulas españolas. International Journal of Psychology an Phychological Therapy, 9(3), 367-378. Disponible en https://www.ijpsy. com/volumen $9 /$ num3/244/bullying-anlisisde-la-situacin-en-las-ES.pdf

Cerezo, F. (2012). Bull-S. Test de Evaluación Socométrica de la Violencia entre Escolares. Manual de Referencia (Versión 2.2). Barakaldo. COHS, Consultores en Ciencias Humanas. Disponible en http://www.gac.com.es/editorial/INFO/ Manuales/bullMANU.pdf

Cerezo, F. (2014). Soporte social del bullying. Análisis de la soledad de la víctima. Revista Electrónica Interuniversitaria de Formación del Profesorado, 17(1), 123-132. Doi: 10.6018/reifop.17.1. 198881

Cerezo, F. (2015). Bullying homofóbico. El papel del profesorado. International Journal of Developmetnal an Educational-Psychology, 1(1), 417-424. Doi: 10.17060/ijodaep.2015.n1.v1.45

Cerezo, F. y Méndez, I. (2012). Conductas de riesgo sociales y de salud en adolescentes. Propuesta de intervención contextualizada para un caso de bullying. Anales de Psicología, 28(3), 705-719. Doi: 10.6018/analesps.28.3.156001
Cerezo, F. y Méndez, I. (2013). Agresores bullying y conductas antisociales. European Journal of Investigation in Healthl, Psichology and Education (EJIHPE), 3(1), 5-14. Doi: 10.30552/ ejihpe.v3i1.32

Cerezo, F., Giménez, A.M., Arnáiz, P. y Prodícimo, E. (2018). Percepción de docentes y estudiantes sobre el ciberacoso: Estrategias de intervención y afrontamiento en Educación Primaria y Secundaria. Revista Cientifica Iberoamericana de Comunicación y Educación, 56, 26-38. Doi: 10.3916/C56-2018-03

Cerezo, F., Sánchez, C., Ruiz, C., y Arense, J.J. (2015). Roles en bullying de adolescente y preadolescentes, y su relación con el clima social y los estilos educativos parentales. Revista de Psicodidáctica, 20(1), 139-155. Doi: 10.1387/ RevPsicodidact.11097

Defensor del Pueblo (2007). Violencia escolar: el maltrato entre iguales en la educación secundaria obligatoria 1999-2006. Madrid: Defensor del Pueblo. Disponible en https://www.defensordelpueblo.es/ wp-content/uploads/2015/05/2007-01-Violencia-escolar-el-maltrato-entre-iguales-en-la-Educaci \% C 3\% B 3n-Secundaria-Obligatoria-1999-2006.pdf

Díaz, MA, Martínez, R. y Martín J. (2010). Estudio estatal sobre convivencia escolar en la Educación Secundaria Obligatoria. Secretaria General Técnica. Centro de publicaciones. Ministerio de Educación. Recuperado de https:// sede.educacion.gob.es/publiventa/estudio-estatal-sobre-la-convivencia-escolar-en-la-educacion-secundaria-obligatoria/educacion-secundaria-socializacion $/ 13567$

Díaz, V., Martínez, O., y Pérez, G. (2017). Consecuencias para la salud del bullying o violencia escolar. Educación, Salud y TIC en contextos multicanales: nuevos espacios de intervención. En R. Zapata, Educación, saludy TIC en contextos multiculturales (pp.268-275). Almería: Universidad de Almería.

Ferrer, R., Albaladejo, N., Sánchez, M., Portilla, I., Jordan, O., y Ruiz, N. (2019). Effectivences of the TEI Program for bullying and Ciberbu- 
llying Reduction and School Climate Improvement. International Environmental Research and Public Health, 16(4), 580. Doi: 10.3390/ ijerph16040580.

Garaigordobil, M., Martínez, V., y Machimbarrena, J.M. (2014). Intervención en el bullying y ciberbullying. Evaluación del caso Martín. Revista Psicología Clínica con Niños y Adolescentes, 4(1), 25-32. Disponible en https://dialnet.unirioja. es/servlet/articulo? codigo $=5789316$

García, X., Pérez, A y Nebot, M. (2010). Factores relacionados con el acoso escolar (bullying) entre los adolescentes de Barcelona. Gaceta Sanitaria, 24(2), 103-108. Doi: 10.1016/j. gaceta.2009.09.017

García, M.V., y Ascensio, C. A. (2015). Bullying y violencia escolar: diferencias, similitudes, actores, consecuencias y origen. Revista Intercontinental de Psicología y Educación, 17(2), 9-38. Disponible en https://www.redalyc.org/articulo.oa? $\mathrm{id}=80247939002$

Giménez, A. M., Arnaiz, P., Cerezo, F., y Prodócimo, E. (2018). Percepción de docentes y estudiantes sobre el ciberacoso. Estrategias de intervención y afrontamiento en Educación Primaria y Secundaria. Comunicar, 26(56), 29-38.

Goleman, D. (1995) Educación emocional. Barcelona: Kairos.

González, A. (2015). Programa TEI “Tutoría Entre Iguales”. Innovación Educativa, 25, 17-32. Disponible en http://www.usc.es/revistas/index. php/ie/article/view/2854/3237

Martínez, P (2017). Maltrato entre iguales en Euskadi 2016. ISEI IVEIInstituto Vasco de Evaluación e investigación Educativa. Bilbao, España: ISEIIVEI. Disponible en https://www.berrigasteiz. com/monografikoak/bizikasi/6002017001c_ Pub_ISEI_tratu_txarrak_2016_c.pdf

Méndez, I. y Cerezo, F. (2010). Test Bull-S: programa informático de evaluación de la agresividad entre escolares. 25 años de integración escolar en Espana: Tecnología e Inclusión en el ámbito educativos, laboral y comunicativo. Murcia: Consejería de Educación, Formación y Empleo. Disponible en https://diversidad.murciaeduca.es/tecnoneet/ 2010/docs/imendez.pdf
Musalem, R. y Castro, P. (2014). Qué se sabe de bullying. Revista Medicina Clínica Condes, 26(1), 14-23. Disponible en https://www.researchgate. net/publication/273834605_Que_se_sabe_ de_bullying

Oñate, A. y Piñuel, I. (2007) Informe Cisneros X: Acoso y violencia escolar en España. Instituto de Innovación Educativa y Desarrollo Directivo. Disponible en http://www.acosoescolar.com/ estudios-cisneros-acoso-escolar/

Olweus, D. (1993). Bullying at school: What we know and what we can do. Cambridge, MA: Blackwell Publishers.

Organización de Naciones Unidas para la Educación, la Ciencia y la Cultura (2018). Nuevos datos revelan que en el mundo uno de cada tres adolescentes sufre acoso escolar. Disponible en https://es.unesco. org/news/nuevos-datos-revelan-que-mundo-cada-tres-adolescentes-sufre-acoso-escolar

Ovejero, A. (2010). Psicología Social: Algunas claves para entender la conducta humana. España: Biblioteca Nueva.

Ovejero, A. Smith, P.K. y Yubero, S (2013). El acoso escolar y su prevención: Perspectivas internacionales. Madrid: Biblioteca Nueva Universidad.

Puértolas, A. y Montiel, I. (2017). Bullying en la educación secundaria: una revisión sobre las características de las víctimas y las víctimas-acosadores y las consecuencias de su victimización. Revista Victimología, 10(5) 85-128. Doi: 10.12827/ RVJV.5.04

Rodríguez, A. y Mejía, Y. (2012). Bullying: un fenómeno por transformar. Duarzary: Revista Internacional de Ciencias de la Salud, 9(1), 98-104. Disponible en https://dialnet.unirioja.es/servlet/ articulo? codigo $=47294204$

Salovey, P., y Mayer, J. D. (1990). Emotional intelligence. Imagination, cognition and personality, 9(3), 185-211. https://doi.org/10.2190/ DUGG-P24E-52WK-6CDG

Sánchez, C. y Cerezo, F. (2011). Estatus social de los sujetos implicados en bullying. Elementos para la mejora de la convivencia en el aula. Revista Española de Orientación y Psicopedagogía, 22(2), 137-149. Recuperado de https://www.redalyc. org/html/3382/338230788004/ 
Sarasola, M. y Ripoll, J.C. (2019). Una revisión de la eficacia de los programas anti-bullying en España. Pulso, 42, 51-72.

Seligman, M. E. P. (1990). Learned Optimism. New York: Knopf.

Solberg, M. E., Olweus, D. y Endresen, I. M. (2007). Bullies and victims at school: Are they the same pupils? British Journal of Educational Psychology, 77(2), 441-464. Doi: 10.1348/000709906X105689

UNESCO. (2019). Behind the numbers: Ending school violence and bullying. Francia: Save the Children. Disponible en https://es.unesco.org/ news/violencia-y-acoso-escolares-son-problema-mundial-segun-nuevo-informe-unesco

Universitat de Barcelona (2017, 3 de julio). Noticias: Andrés González: "Con el programa TEI, desaparece en un $95 \%$ el acoso escolar, se minimiza la victimización y se disminuye el nivel de estrés y de ansiedad". Barcelona. Universitat de Barcelona. Recuperado de https:/www.ub.edu/web/ ub/es/menu_eines/noticies/2017/entrevistes/ agonzalez.html
Vanega, S., Sosa, M. y Castillo, R. (2018). Acoso escolar, ira y depresión en adolescentes mexicanos: un estudio preliminar de la eficacia de una intervención. Revista de Psicología Clínica con Niños y Adolescentes, 5(2), 49-54.

Vieira, M., Fernández, I. y Quevedo, G. (1989). Violence, Bullying and counseling in the Iberian Península. En E. Roland y E. Munthe. Bulling an International Perspective (p. 35-52). Londres: Routledge. 